\title{
Ecological strategies in stable and disturbed environments depend on species specialisation
}

\author{
Lucie Büchi and Séverine Vuilleumier
}

L. Büchi (http://orcid.org/0000-0002-1935-6176)(lucie.buchi@gmail.com) and S. Vuilleumier, Dept of Ecology and Evolution, and Inst. of Microbiology, Univ. of Lausanne, CH-1015 Lausanne, Switzerland. Present address for LB: Agroscope, Inst. for Plant Production Sciences, CH-1260 Nyon, Switzerland. SV also at: School of Life Sciences, Ecole Polytechnique Fédérale de Lausanne, CH-1015 Lausanne, Switzerland.

\begin{abstract}
Ecological strategies are integral to understanding species survival in different environments. However, how habitat specialisation is involved in such strategies is not fully understood, particularly in heterogeneous and disturbed environments. Here, we studied the trait associations between specialisation, dispersal ability, competitiveness, reproductive investment and survival rate in a spatially explicit metacommunity model under various disturbance rates. Though no unique trait values were associated with specialisation, relationships were uncovered depending on environmental factors. We found strong trait associations mainly for generalist species, while specialist species exhibited a larger range of trait combinations. Trait associations were driven first by the disturbance rate and second by species' dispersal ability and generation overlap. With disturbance, low dispersal ability was strongly selected against, for specialists as well as for generalists. Our results demonstrate that habitat specialisation is critical for the emergence of trait strategies in metacommunities and that disturbance in interaction with dispersal ability limits not only the range of trait values but also the type of possible trait associations.
\end{abstract}

Characteristics such as size, dispersal ability, reproduction, or survival rate, vary drastically among species. For example, the magnitude of divergence of seed size and mass among flowering plants can reach $10^{11}$ and $10^{5}$, respectively (Westoby et al. 1992). Similarly, flowering plants' longevity ranges from desert annuals, completing their life cycle in a few weeks, to thousand-year-old trees such as the bristlecone pine Pinus longaeva (Borges 2009).

These variations in life history or functional trait values are shaped by the evolutionary history of the species; for a given species, the association of particular trait values defines its ecological strategy, or syndrome (Westoby 1998, Reich et al. 2003, Grime and Pierce 2012). A strategy is the result of selection for trait values whose combination confers a higher fitness in a given environment (Agrawal et al. 2010, Stevens et al. 2014). This selection can act on independent traits, or on traits constrained by tradeoffs, narrowing the range of possible trait combinations.

Ecological strategies have been thoroughly studied and several frameworks focusing therein have been developed, from the classical $\mathrm{r} / \mathrm{K}$ selection theory (MacArthur and Wilson 1967, Pianka 1970) and Grime CSR classification for vascular plants (Grime 1977), to the more recent leafheight-seed strategy scheme and leaf economics spectrum (Westoby 1998, Westoby et al. 2002, Wright et al. 2004, Adler et al. 2014, Reich 2014). The description of species' ecological strategies is intimately linked to their environment, especially in terms of the intensity of stress and disturbance experienced (Grime 1977, Southwood 1988, Grime and Pierce 2012, Westoby 1998).

Habitat specialisation also varies among species: at one extreme, specialists exhibit a narrow niche breadth or environmental tolerance while generalists have a larger breadth or tolerance (Levins 1968, Futuyma and Moreno 1988, Poisot et al. 2011). This degree of specialisation changes how species experience their environments; for example, a given environment may be perceived as more homogeneous by a generalist species than by a specialist one. As a consequence, specialisation may also influence the selection on trait values underlying ecological strategies. Despite the likelihood of such influence, few studies have investigated these links specifically. Most studies have focused on the relationship between specialisation and dispersal. Empirically, specialists generally have a low dispersal rate (Fig. 1A) (Warren et al. 2001, Verberk et al. 2010). Large scale empirical studies in European land snails (Dahirel et al. 2015) and in European birds (Reif et al. 2015) also tended to associate specialist species with low dispersal. Despite this, specialisation has paradoxically been associated with high dispersal abilities (Fig. 1B), particularly when specialisation concerns sparse or ephemeral habitats (Levin and Muller-Landau 2000, Fridley et al. 2007). Stevens et al. (2014) have also suggested that while a link between specialisation and dispersal is expected, it is difficult to predict the direction of this correlation. 
(A)
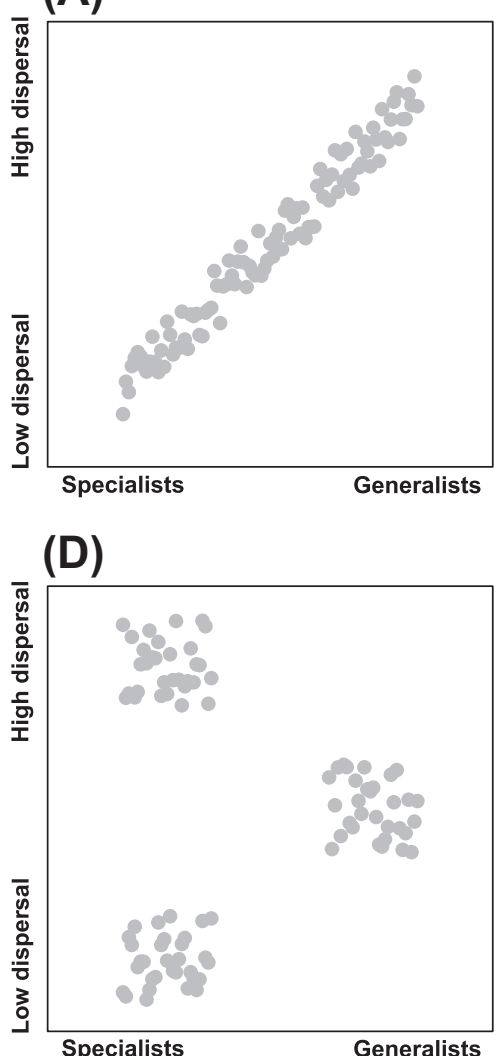

(B)

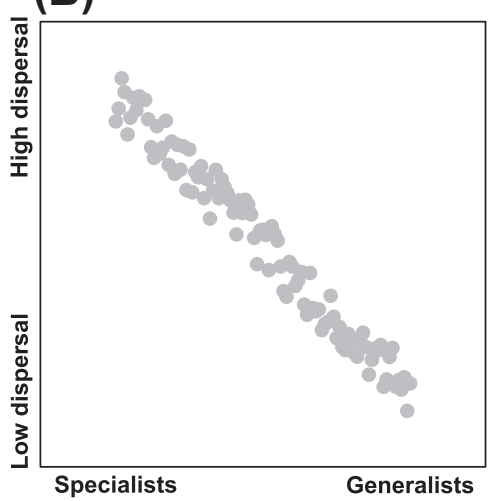

(E)

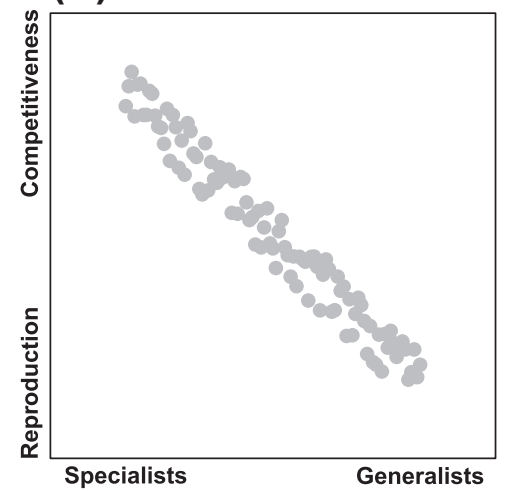

(C)

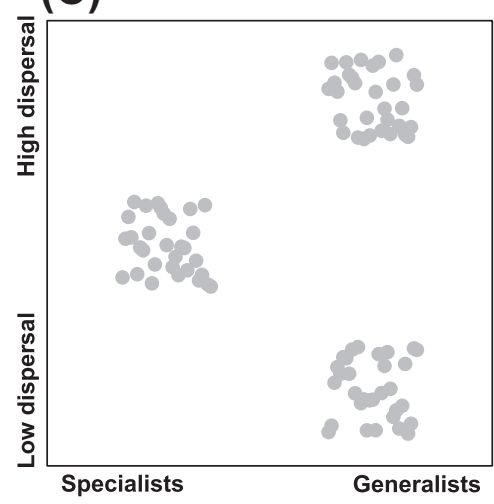

(F)

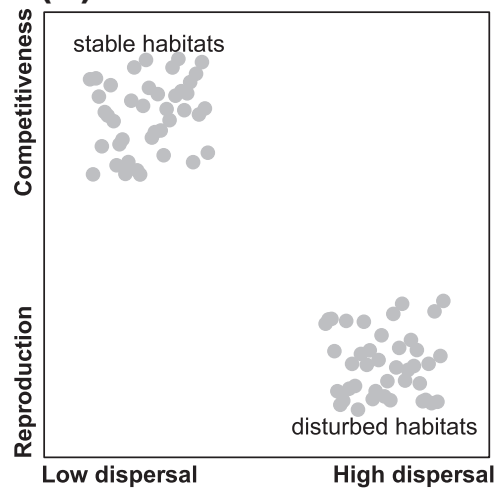

Figure 1. Expectations for trait strategies from different empirical and theoretical studies. (A), (B), (C) and (D): relationship between dispersal and niche breadth, (E): relationship between competitiveness (in tradeoff with reproductive investment) and niche breadth, $(\mathrm{F})$ : relationship between competitiveness and dispersal, in stable and disturbed habitats.

Theoretically, studies have generally shown that generalist species are commonly associated with disturbed and heterogeneous environments, and specialist species with stable and homogeneous habitats (Futuyma and Moreno 1988, Devictor et al. 2008). In parallel, investigations about the coevolution of life history traits have suggested that low rates of dispersal are required for specialisation to evolve (Brown and Pavlovic 1992, Kisdi 2002, Ravigné et al. 2009, Débarre and Gandon 2010, Nurmi and Parvinen 2011). Other studies have suggested that specialists could be favoured at intermediate dispersal rates (Fig. 1C) (Ronce and Kirkpatrick 2001) or, inversely, that they can be advantaged by small or large values (Fig. 1D) (Nurmi and Parvinen 2008). However, analysing the conditions required for specialisation to evolve is not equivalent to investigating the traits leading to the most efficient strategies, especially in changing environments. The lack of consensus on the direction of covariation between dispersal ability and specialisation may well be due to the multiple roles of dispersal seen in life-history strategies (Poisot et al. 2011, Stevens et al. 2014), as well as different dispersal attributes (rate, distance and temporality) and phases (Bonte et al. 2012, Buoro and Carlson 2014).

Less evidence has been shown regarding the links between specialisation and other life history traits. Reif et al. (2015) have revealed complex relationships between habitat specialisation, diet and climatic niches and flight attributes. Specialists are also traditionally associated with K-strategy characteristics (Southwood 1988), such as high competitive ability (Fig. 1E,F) (Marvier et al. 2004). Specialists may also have longer life spans than generalists (Fridley et al. 2007). These examples demonstrate that there is little consensus on the association between the degree of specialisation and other traits, and that these aspects deserve further investigations.

Most of the theoretical studies investigating the coevolution of life history traits and ecological strategies include few habitat types and few competing species, mainly due to difficult analytical tractability. Moreover, important life history traits other than dispersal, such as survival rate or competitiveness have seldom been considered. Understanding the evolution of specialisation and related strategies therefore deserves investigation in wider settings. A simulation approach considers the multiplicity and complexity of trait interactions involved in building ecological strategies. In this study, we use simulation experiments within spatially explicit environments to investigate the selection of strategies in metacommunities under different disturbance regimes. The modelling approach mimics community assembly processes. Each simulation starts with the creation of a large pool of species harbouring different strategies, which are then selected by environmental and spatial processes. Strategies combine different values of habitat specialisation and dispersal ability, competitiveness and reproductive investment. We analyse the resulting trait value associations across different values of generation overlap and disturbance. We expect that different degrees of specialisation are 
associated with distinct strategies. In particular, we investigate the relationship between specialisation and dispersal and whether generalist species show trait attributes linked to opportunistic strategies such as low competitiveness (Fig. 1). We predict, by modifying the intensity of competition and the spatial distribution of available habitats, that both disturbance rate and generation overlap impact the trait associations observed.

\section{Methods}

\section{Model description}

We used a metacommunity simulation model in which trophically-equivalent species (e.g. all plants) compete for space in a heterogeneous environment (Büchi et al. 2009, Büchi and Vuilleumier 2014). This model simulates sessile species, in which only juveniles disperse prior to settlement. The environment is composed of $25 \times 25$ local communities, or cells, each cell being characterized by a carrying capacity $K$ of 100 individuals and an environmental value $E_{i}$ that determines species growth rate. $E_{i}$ varies from cell to cell (heterogeneous environment) and follows a normalized gaussian probability distribution (with mean $=0$ and standard deviation $=1$, Büchi et al. 2009). Metacommunity dynamics proceed in four discrete time steps: 1) reproduction, 2) adult mortality and disturbance, 3) juvenile dispersal and 4) competition for space. Each species is characterized by six traits: niche optimum $\mu_{s}$, niche breadth $\sigma_{s}$, dispersal ability $\delta_{s}$, competitiveness $\kappa_{s}$, reproductive investment $\omega_{s}$ and survival rate $\psi_{s}$.

Species' habitat specialisation impacts their fecundity, with specialist species having a higher fecundity in their optimal habitat compared to generalist species. Species fecundity $R_{s}$ in a community (cell) of environmental value $E_{i}$ is determined by the individual reproductive investment $\omega_{s}$, niche optimum $\mu_{s}$, and niche breadth $\sigma_{s}$ as follows:

$$
R_{s}\left(E_{i}\right)=h \times \frac{\omega_{s}}{\sigma_{s} \sqrt{2 \pi}} \times\left[-\frac{1}{2} \times\left(\frac{E_{i}-\mu_{s}}{\sigma_{s}}\right)^{2}\right]
$$

Here $h$ is a scaling factor transforming the rate of increase into an effective fecundity ( $h$ is set to 10 in this study). The effective per capita number of offspring in each cell is the rounded value of $R_{s}\left(E_{i}\right)$.

Mortality occurs after reproduction and is determined by adult survival $\psi_{s}$ and disturbance rate $T$. Adult survival probability equals to $\psi_{s}$ at each time step. Disturbance causes total extinction in proportion $T$ of randomly chosen local communities (cells).

Juvenile dispersal occurs according to a dispersal kernel $D_{s}(x)$ (probability density function of dispersal distance) whose shape is defined by the mean species dispersal ability $\delta_{s}$,

$$
D_{s}(x)=\frac{1}{\delta_{s}} \times \exp \left[-\frac{x}{\delta_{s}}\right]
$$

Each juvenile disperses independently at a distance that is determined by the dispersal kernel associated with its species. Direction is drawn randomly from a uniform distribution. Periodic boundary conditions are considered, such that individuals reaching the environment borders re-enter the environments from the opposite side.

Preemptive competition is assumed (i.e. adult individuals already settled cannot be displaced by juveniles). Therefore, only juveniles compete for settlement in each local community; successful juveniles are drawn randomly among the pool of competing juveniles, with a weighting determined by their competitiveness $\kappa_{s}$. If there are fewer competing juveniles than the number of places available, all juveniles can settle.

We further assume that competitiveness $\kappa_{s}$ and reproductive investment $\omega_{s}$ are constrained by the following tradeoff (Levine and Rees 2002):

$\kappa_{s} \times \omega_{s}=1$

This tradeoff is inspired by the classic, well-documented tradeoff between seed size and seed number (Jakobsson and Eriksson 2000, Leishman 2001).

In this model, traits are randomly associated in species and advantageous strategies are selected when providing a fitness advantage to species. During this process, trait values remain constant. We thus assume that the time scale of the processes studied does not allow for the evolution of traits, and only community dynamics are considered.

This model is an object-oriented model implemented in Borland Delphi, and code is available from the Dryad Digital Repository: <http://dx.doi.org/10.5061/dryad. ms738> (Büchi and Vuilleumier 2015).

\section{Simulations}

For each simulation, we generated an initial metacommunity composed of species to which we assigned trait values as described in Table 1. Three distinct scenarios were built in order to address the influence of the different species traits sequentially. In the first scenario, all species had competitiveness $\kappa_{s}$ and reproductive investment $\omega_{s}$ set to 1 and had variable dispersal ability $\delta_{s}$ drawn randomly between 0 and 1 . In the second scenario, all species had variable competitiveness $\kappa_{s}$ and reproductive investment $\omega_{s}$, which were randomly drawn for each species following the tradeoff presented in Eq. 3 (uniform distribution of their logarithms), and had an identical and limited dispersal ability $\delta_{s}$ set to 0.1 . In the third scenario, all species had variable dispersal ability, competitiveness and reproductive investment. In all scenarios, we varied for each species niche optimum $\mu_{s}$ (values between -2.5 and 2.5) and niche breadth $\sigma_{s}$ (values between 0.01 and 1). A value of 0.01 for $\sigma_{s}$ corresponded to very specialist species and a value of 1 corresponded to

Table 1. Values of the parameters used in the simulations. In bold the parameters varying between simulations.

\begin{tabular}{lcl}
\hline Parameters & Symbols & \multicolumn{1}{c}{ Values } \\
\hline Niche optimum & $\mu_{s}$ & random $[-2.5-2.5]$ \\
Niche breadth & $\sigma_{s}$ & random $[0.01-1]$ \\
Competitiveness & $\boldsymbol{\kappa}_{s}$ & $\mathbf{1} ;$ random* $[\mathbf{0 . 1}-\mathbf{1 0}]$ \\
Reproductive investment & $\boldsymbol{\omega}_{s}$ & $\mathbf{1} ;$ random* $[\mathbf{0 . 1}-\mathbf{1 0}]$ \\
Dispersal ability & $\boldsymbol{\delta}_{s}$ & $\mathbf{0 . 1} ;$ random $[\mathbf{0}-\mathbf{1}]$ \\
Survival rate & $\boldsymbol{\psi}_{s}$ & $\mathbf{0 . 9 5} ; \mathbf{0}$ \\
Disturbance rate & $\boldsymbol{T}$ & $\mathbf{0} ; \mathbf{0 . 0 1} ; \mathbf{0 . 2 5}$ \\
\hline
\end{tabular}

*tradeoff: $\kappa_{\mathrm{s}} \times \omega_{\mathrm{s}}=1$. 
very generalist species. Then, for each scenario, we investigated the influence of generation overlap (adult annual survival) and disturbance as follows. Each scenario was run alternatively with adult annual survival rate $\psi_{s}$ set to either 0 (no generation overlap) or to 0.95 (strong generation overlap). Three rates of disturbance $T$ were considered: $T=0, T=0.01$ and $T=0.25$.

For each case studied, we considered pools of 100 different species to initiate the simulations. Individuals of these species were randomly distributed in the landscape until carrying capacity was reached in each cell. Metacommunity dynamics were simulated for 1000 time steps, during which some species went extinct and others persisted. This duration is sufficient to reach a steady state in terms of mean traits (weighted by species abundances) though the species richness continued to decrease very slowly (Supplementary material Appendix 1). For each of the simulation scenarios, 50 replicates were run, with a new species pool and landscape for each replicate. At the end of each simulation, we recorded the characteristics and abundance of the selected strategies in terms of niche breath, dispersal ability, competitiveness and reproductive investment. Mean trait values were computed for each simulation considering the trait values of all the surviving individuals (mean species trait value weighted by the abundance of each species). Results for trait associations given niche breadth were analysed for five categories of niche breadth (from the most specialist to the most generalist species): $0-0.1,0.1-0.2,0.2-0.4,0.4-0.6,0.6-1$.

We also investigated the sensitivity of our results to the initial number of species (10 or 1000) and to different environmental spatial autocorrelation. To generate autocorrelation between the environmental values of two cells, we used the sequential Gaussian simulation algorithm (Goovaerts 1998, see also Fig. 1 in Büchi et al. 2009). We considered moderate $(\alpha=5)$ and strong $(\alpha=10)$ autocorrelation, where $\alpha$ represents the distance above which correlation falls below 0.05 ( $\alpha=0$ in the other simulations). These results are presented in Supplementary material Appendix 2 and 3.

Simulations results were analysed using R 3.0.2 (<www.rproject.org $>$ ).

\section{Data deposition}

Data available from the Dryad Digital Repository: <http:// dx.doi.org/10.5061/dryad.ms738> (Büchi and Vuilleumier 2015).

\section{Results}

\section{Associations between niche breadth and dispersal when competitiveness and reproductive investment are fixed}

Metacommunities are mainly composed of species with low values of niche breadth, namely specialist species (Fig. 2). Interestingly, a strong association between niche breadth and dispersal abilities is observed (Fig. 2). In the absence of disturbance $(T=0)$, communities are composed of a high number of specialist species with a large range of dispersal abilities, but with very low abundances at the lowest dispersal values, while more generalist species have lower dispersal abilities (Fig. 2). The presence of disturbance affects the association between species niche breadth and dispersal abilities, such that specialist and generalist species with limited dispersal values are progressively driven to extinction (Fig. 2). Generation overlap changes this pattern only quantitatively, except at high disturbance rate where the pattern is completely changed. In this situation, mean dispersal is higher than in any other situation, and generalist species have higher dispersal abilities than specialist ones (Fig. 2).

The associations found between niche breadth and dispersal are quantitatively but not qualitatively impacted by spatial autocorrelation (Supplementary material Appendix $3)$. An exception occurs when disturbance is high $(T=0.25)$ and generations overlap. In this case, when spatial autocorrelation is low, dispersal was lower for all species, though this effect was stronger for the most generalist species.

In the absence of disturbance, species can survive with a large range of niche optimum values, whereas species with extreme niche optima (thus relying on rare habitats) disappear in the presence of strong disturbance (Supplementary material Appendix 4).

\section{Associations between niche breadth and competitiveness/reproductive investment when dispersal ability is limited}

In the absence of disturbance $(T=0)$, metacommunities are composed of species with a large range of competiveness and niche breadth, but with a higher proportion of species exhibiting small niche breadth values (Fig. 3). With no generation overlap, generalist species show reduced investment in competitiveness compared to specialist species with a large range of possible competitiveness and a mean value similar to the initial state (Fig. 3). When generations overlap, all categories of specialisation show both a mean and range of values similar to the initial state, with no clear selection of a particular strategy.

Disturbance tends to decrease global investment in competitiveness in favour of reproduction. When disturbance is moderate $(T=0.1)$, the association between competitiveness and niche breadth is still negative, with competitiveness being globally lower with generation overlap. In contrast, at high disturbance rate $(T=0.25)$, all categories of niche breadth show similarly low investment in competition, with a reduced range of viable trait values (Fig. 3). This pattern is particularly strong when generations overlap.

Here again, spatial autocorrelation has a slight quantitative influence on the relationship between niche breadth and competitiveness (Supplementary material Appendix 3). However, when disturbance was high $(T=0.25)$, strong spatial autocorrelation allowed for a larger range of competitiveness values for specialist species.

With limited dispersal ability, very generalist species can survive in the metacommunity. They are however, in the absence of disturbance, restricted to extreme niche optima. In contrast, very generalist species with intermediate niche optimum can survive in the presence of disturbance (Supplementary material Appendix 4). 

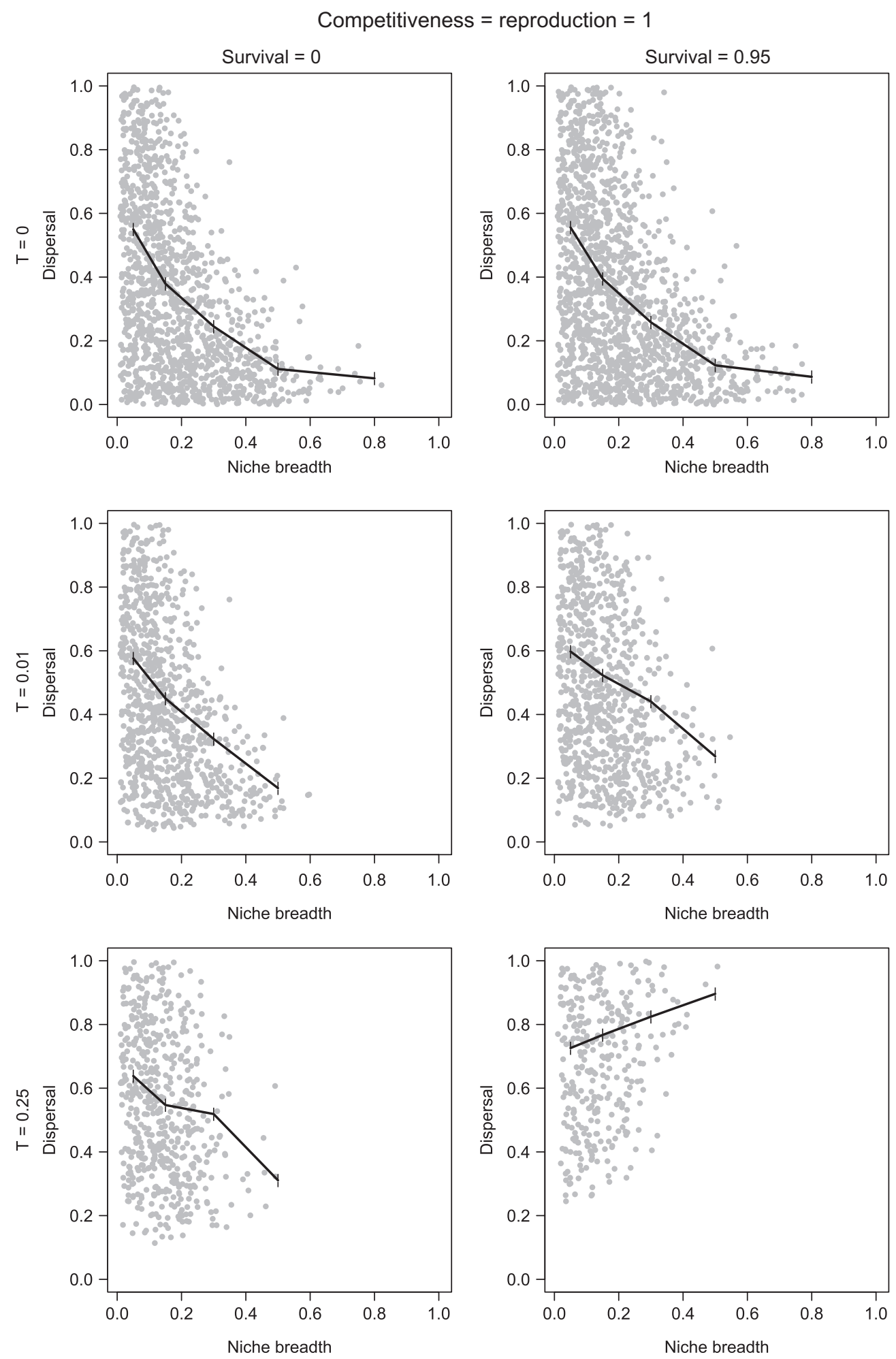

Figure 2. Association between dispersal and niche breadth values in communities experiencing different strength of disturbance: $\mathrm{T}=0$ : upper row, $\mathrm{T}=0.01$ : middle row, $\mathrm{T}=0.25$ : lower row. Results are presented for species with competitiveness and reproductive investment equal to 1 , without and with generation overlap (survival rate at each generation is either 0 or 0.95 ). The grey dots represent the strategies of the surviving species, the black line is the mean dispersal, weighted by species abundances, computed for five categories of niche breadth $(0.0-0.1 ; 0.1-0.2 ; 0.2-0.4 ; 0.4-0.6 ; 0.6-1.0)$. Each graph represents the pooled results over the 50 replicates.

\section{Associations between niche breadth, dispersal and competitiveness/reproductive investment}

The change from fixed values of competitiveness and reproductive investment to variable values has almost no influence on the association between dispersal ability and niche breadth (Fig. 4 versus Fig. 2).

In contrast, the change from fixed values of dispersal ability to variable values has a clear influence on the association between competitiveness and niche breadth (Fig. 5 versus 

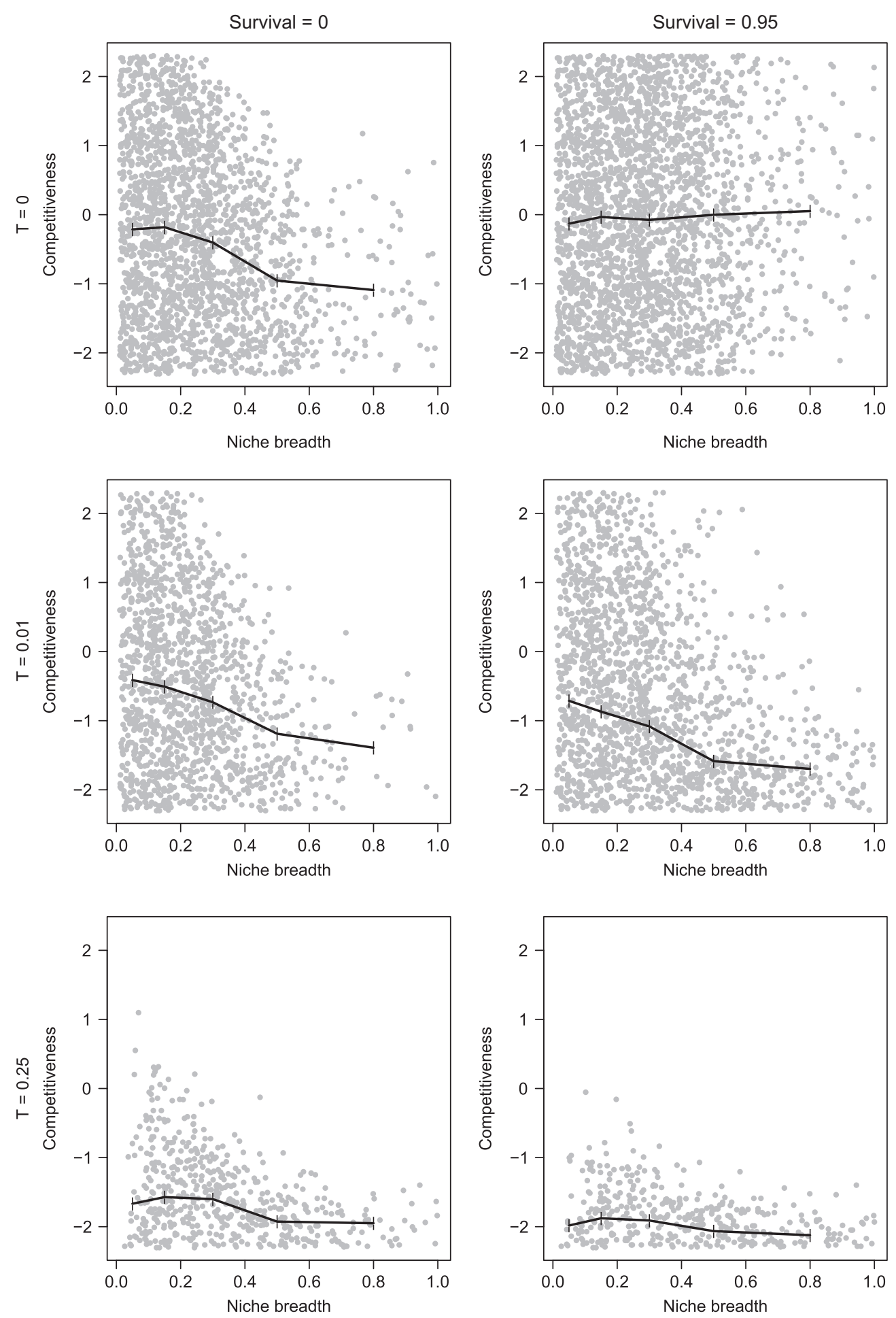

Figure 3. Association between competitiveness (on a logarithmic scale) and niche breadth in communities experiencing different strength of disturbance: $\mathrm{T}=0$ : upper row, $\mathrm{T}=0.01$ : middle row, $\mathrm{T}=0.25$ : lower row. Results are presented for species with limited dispersal, without and with generation overlap (survival rate at each generation is either 0 or 0.95 ). The grey dots represent the strategies of the surviving species, the black line is the mean competitiveness, weighted by species abundances, computed for five categories of niche breadth $(0.0-0.1$; $0.1-0.2 ; 0.2-0.4 ; 0.4-0.6 ; 0.6-1.0)$. Each graph represents the pooled results over the 50 replicates.

Fig. 3). First of all, dispersal limitation allows for the survival of a larger number of generalist species than a variable dispersal ability. Then the decrease of competitiveness with increasing niche breadth is clearly less pronounced when dispersal is variable (Fig. 5). The range of competitiveness values observed in the presence of disturbance is also clearly higher, especially at low niche breadth. So here, under disturbance and depending on dispersal limitation, communities can 

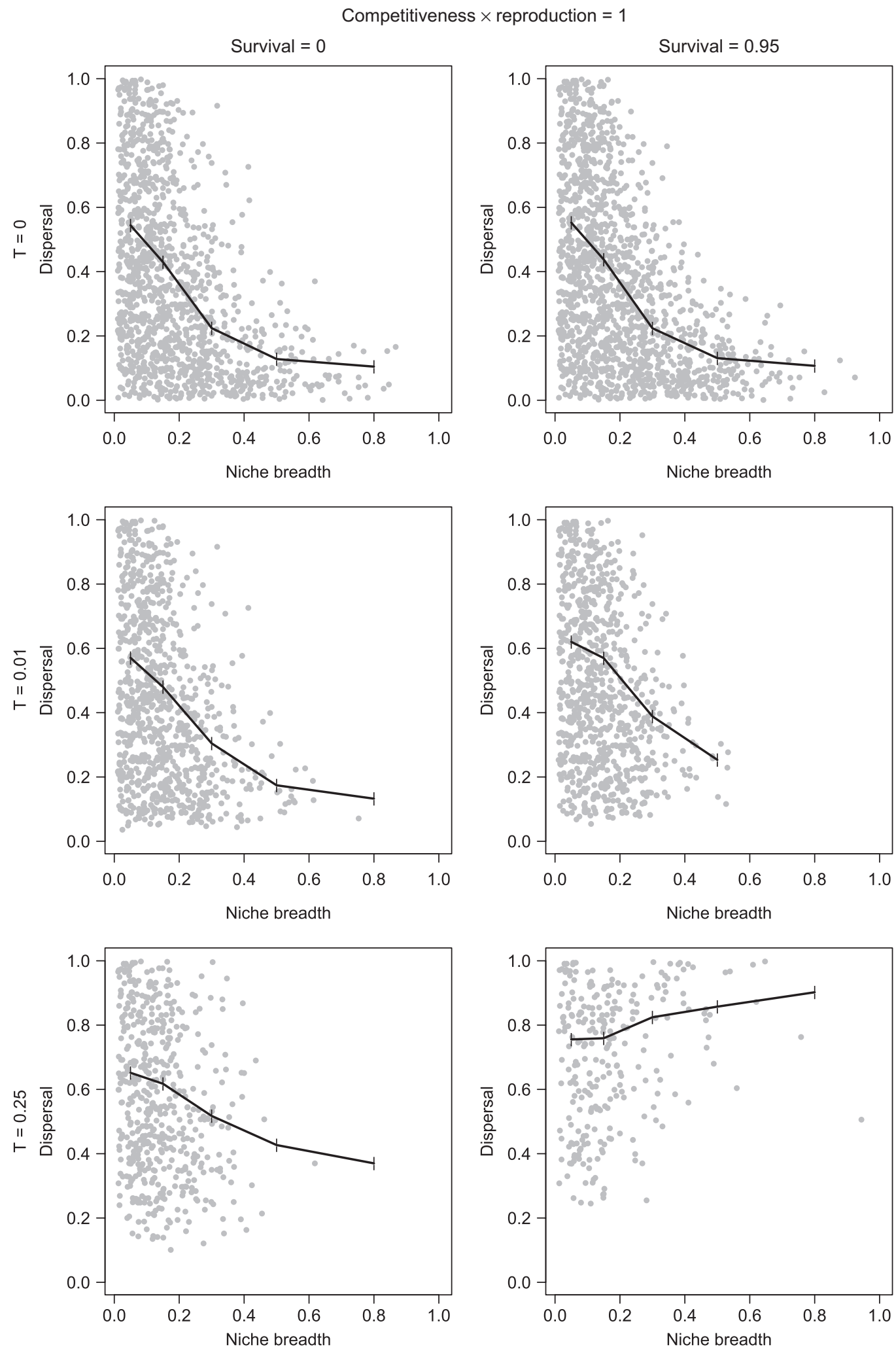

Figure 4. Association between dispersal and niche breadth values in communities experiencing different strength of disturbance: $\mathrm{T}=0$ : upper row, $\mathrm{T}=0.01$ : middle row, $\mathrm{T}=0.25$ : lower row. Results are presented for species with variable values of competitiveness and reproductive investment, without and with generation overlap (survival rate at each generation is either 0 or 0.95 ). The grey dots represent the strategies of the surviving species, the black line is the mean dispersal, weighted by species abundances, computed for five categories of niche breadth $(0.0-0.1 ; 0.1-0.2 ; 0.2-0.4 ; 0.4-0.6 ; 0.6-1.0)$. Each graph represents the pooled results over the 50 replicates. 

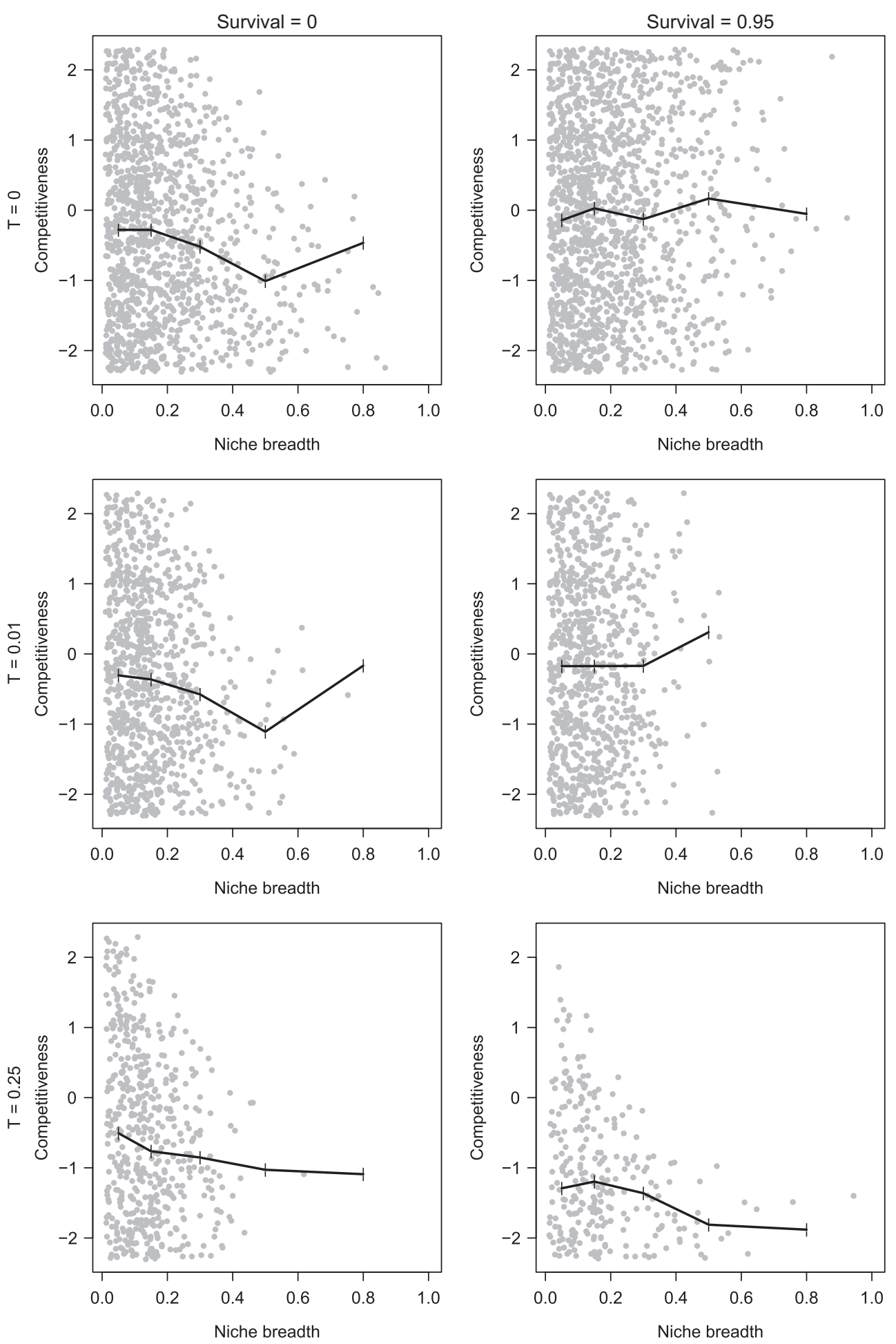

Figure 5. Association between competitiveness (on a logarithmic scale) and niche breadth values in communities experiencing different strength of disturbance: $\mathrm{T}=0$ : upper row, $\mathrm{T}=0.01$ : middle row, $\mathrm{T}=0.25$ : lower row. Results are presented for species with randomly assigned dispersal values, with and without generation overlap (survival rate at each generation is either 0 or 0.95 ). The grey dots represent the strategies of the surviving species, the black line is the mean competitiveness, weighted by species abundances, computed for five categories of niche breadth $(0.0-0.1 ; 0.1-0.2 ; 0.2-0.4 ; 0.4-0.6 ; 0.6-1.0)$. Each graph represents the pooled results over the 50 replicates.

be either composed by specialist species with a broad range of competitiveness/reproductive investment values or by generalist and specialist species that invest in reproduction and limit their investment in competitiveness.
Associations between dispersal and competitiveness/ reproductive investment are visible when taking into account the niche breadths of the surviving species, especially at high disturbance rates (Fig. 6). When none or moderate 
disturbance occurs and there is no generation overlap, generalists associate higher reproductive investment with lower dispersal ability compared to specialists. When no disturbance occurs and generation overlap is high, generalists have lower dispersal ability but with no clear selection on competitiveness values. The pattern changes when disturbance rate is strong and generation overlap is high. In this case, generalist species have higher dispersal ability with higher reproductive investment than specialist species (Fig. 6).

\section{Discussion}

In this study, we delineated ecological strategies by level of specialisation. We found larger ranges and more numerous combinations of trait values for specialist species, but more restricted trait combinations for the most generalist species. Additionally, our results show that species specialisation and trait associations in metacommunities strongly depend on the intensity of disturbance experienced by the species. Then, within a disturbance rate, species' ability to disperse and generation overlap act in interaction on the trait associations observed.
Most of our results can be explained by the mechanisms that determine species' success in settlement. In their optimal habitat, specialist species are dominant over generalists and tend to exclude them, as they produce more juveniles. However, at the metacommunity scale, specialist species, due to their specific habitat requirements, suffer from limited available habitat with a scattered distribution. Their dispersal ability thus determines the amount of suitable habitat accessible. Under disturbance, extinction-colonization dynamics favour species that disperse (but see Ronce et al. 2000a); however, specialists succeed in settling in their optimal habitat only when dispersal is very high. In contrast, generalist species, thanks to their high environmental tolerance, benefit from a large number of suitable habitats, accessible without large dispersal abilities. Generalists therefore have the advantage of easy settlement over specialists when dispersal is limited. In contrast, while specialist species' persistence is limited by their dispersal ability, generalist species' persistence is limited instead by their low fecundity. Finally, the effective amount of habitat available for settlement is regulated by adult survival (generation overlap) and disturbance (local community extinction). Combinations of these processes drive the composition of metacommunities and the surviving strategies observed in our study.

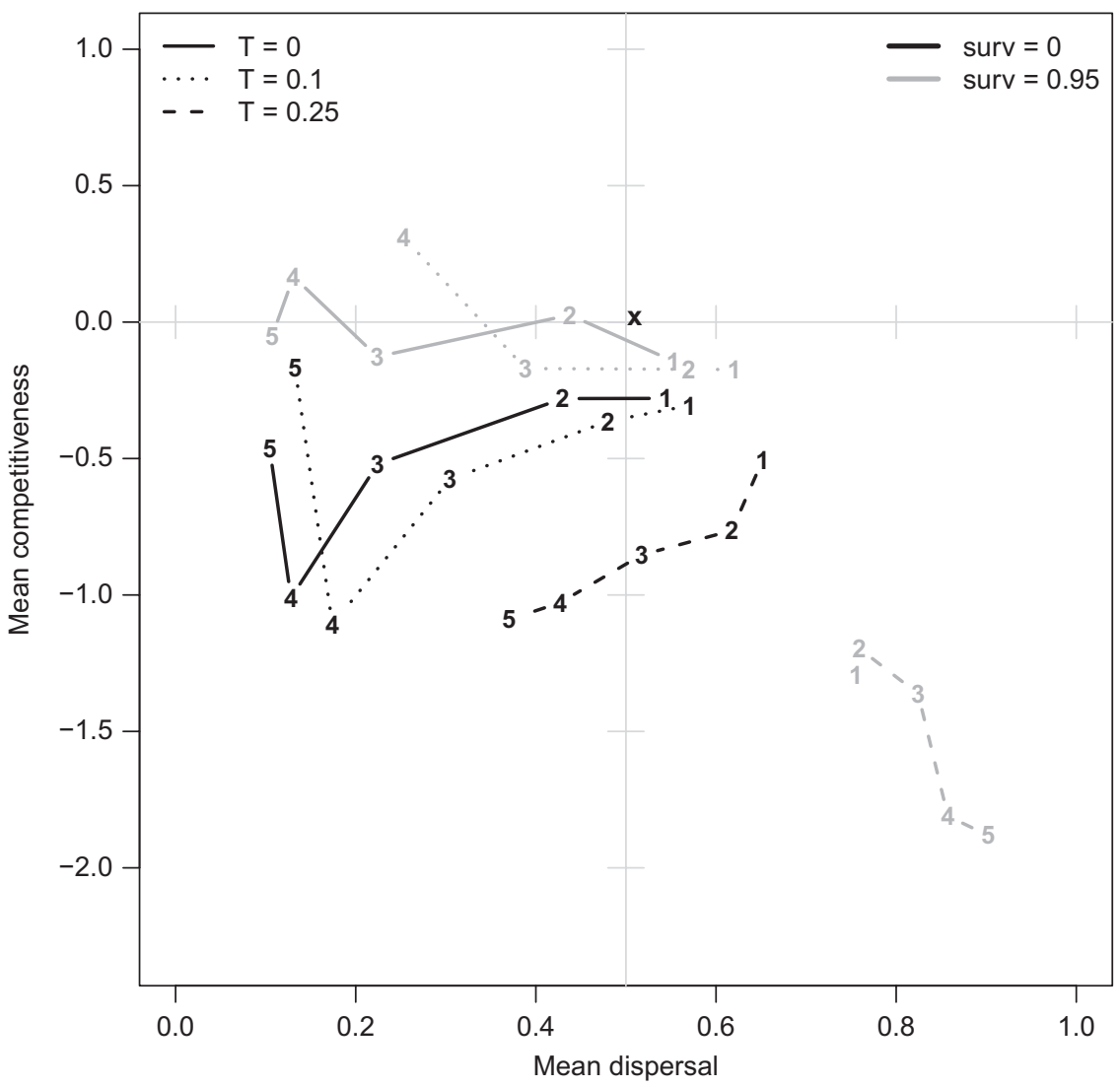

Figure 6. Association between dispersal ability and competitiveness (log values) for five categories of niche breadth. The mean values are the average over the 50 replicates of the values observed in all surviving individuals. The numbers correspond to the five niche breadth categories from the most specialist species to the most generalist species: $1: 0.0-0.1 ; 2: 0.1-0.2 ; 3: 0.2-0.4 ; 4: 0.4-0.6$; $5: 0.6-1.0$. The cross shows the mean values of the metacommunity at the initial stage of the simulations. Plain lines: no disturbance $(T=0)$, dotted lines: intermediate disturbance rate $(T=0.01)$, dashed lines: high disturbance rate $(T=0.25)$. In black, non-overlapping generations $\left(\right.$ survival rate $\left.\psi_{s}=0\right)$ and in grey overlapping generations (survival rate $\psi_{s}=0.95$ ). 


\section{Strategies involving specialisation and dispersal}

Strategies involving specialisation and dispersal abilities are dependent on the disturbance rate. In the absence of disturbance, our results show that generalist species have limited dispersal ability, while in contrast, specialist species are associated with a large range of dispersal abilities. These results can be explained first by the cost of dispersal and second by the amount of suitable habitat accessible. In the absence of disturbance, dispersal is costly because the probability of a juvenile reaching an unsuitable habitat is high (Bonte et al. 2012), and thus limited dispersal abilities develop (Hastings 1983). This result is observed here for generalist species but not for specialist species. Indeed, specialist species produce more juveniles than the number of local settlement opportunities; dispersal therefore favours their persistence despite its inherent cost by allowing them to reach new habitats. In addition, limited dispersal ability drastically reduces specialist species' chances to settle in their specific, rare and scattered suitable habitat. This result is reflected in the lower abundance observed for the most specialist species at very low dispersal abilities. In contrast, generalist species are less impacted by the distribution of their habitats, which tend to be widely distributed and could be easily reached through dispersal from neighbouring habitats (Nilsson et al. 1993, Dynesius and Jansson 2000).

The presence of disturbance strongly modifies the association between species niche breadth and dispersal abilities. Under disturbance, specialist and generalist species with limited dispersal abilities are excluded, as disturbance induces extinction-colonization dynamics favouring species with high dispersal (Gandon and Michalakis 1999). Generalists face additional barriers as well, such as their very low growth rate, which does not withstand frequent disturbance (Parvinen and Egas 2004, Nurmi and Parvinen 2008). The coexistence of specialist and generalist species is also driven by generation overlap. When adult survival is null, recruitment to suitable habitat is high and specialist species dominate over generalist species as they produce more juveniles. In contrast, when generations strongly overlap, saturation of the metacommunity is very high. In such a saturated system, disturbance creates gaps in adult density, and thus many spots are available locally for juveniles. In this case, species with high dispersal ability have a strong advantage in finding these newly opened positions. The production of numerous offspring can confer similar advantages, explaining why very fecund specialists can perform well even when they have lower dispersal ability. Some examples are tropical forests or coral reefs, where sudden gaps are often the only way for new recruitment to occur (Connell 1978).

These results contrast the classical descriptions of specialists as having reduced dispersal ability and generalists as being opportunistic species with high dispersal ability. This classic view is supported by theoretical studies on the evolution of specialisation, or local adaptation, in interaction with dispersal (Brown and Pavlovic 1992, Kisdi 2002, Ravigné et al. 2009). Those studies demonstrate that reduced dispersal is required for specialisation, as dispersal prevents adaptation to a local environment. As a corollary, high dispersal promotes generalist species by increasing the variety of habitats a species experiences. The discrepancy between these classic descriptions and the results seen here can be explained by differences in the processes studied. Here, we investigated the dynamics and factors that drive specialist-generalist coexistence in metacommunities and not how specialisation itself might evolve (e.g. from an initial pool of generalist species). Although dispersal limitation favours specialists, there is still a need to disperse in order to persist in dynamic and heterogeneous environments. Interestingly, the diversity of trait associations observed in our study is reflected by some empirical evidence. Both generalist and specialist species can have variable dispersal abilities (Nilsson et al. 1993, Levin and Muller-Landau 2000, Warren et al. 2001, Verberk et al. 2010).

\section{Strategies involving specialisation and competitiveness/reproductive investment}

Strategies involving specialisation and competitiveness (or reproductive investment) are less obvious than those involving dispersal, and are dependent on the disturbance rate in interaction with generation overlap and dispersal ability. In the absence of disturbance and when generation overlap is strong, recruitment is low and the coexistence of specialist and generalist species is independent of their investment in competition or reproduction. Forests are a typical example of a natural ecosystem with high generation overlap, as they are mainly composed of long-lived species. In contrast, without generation overlap, recruitment is high and generalists investing in competition rather than reproduction are excluded by specialist species (Nurmi and Parvinen 2008). Specialist species, which produce many juveniles in their given habitat, can afford to invest more in competitive ability, while generalist species are strongly limited by their reduced fecundity (Nurmi and Parvinen 2008).

Disturbance has a drastic impact on the associations between niche breadth and competitiveness or reproductive investment, but the patterns depend strongly on the dispersal ability of the species. When dispersal is unconstrained, communities are composed of specialist species, with generalists excluded. Disturbance creates extinction-colonization dynamics that are known to favour dispersal, and provides empty habitat patches that are efficiently colonized by specialist species due to two factors: their local fitness advantage and the larger number of dispersing juveniles. Thus, disturbance favours species with high dispersal and fecundity as they efficiently settle in empty habitat patches. Dispersal limitation, however, completely shifts this pattern: though new habitat becomes available after disturbance, the sparseness of suitable habitat for specialists and their limited ability to disperse prevents occupation of these new areas, and thus prevents displacement of the (less efficient) generalists. Communities are composed of species with a wide range of specialisation but usually with all species investing in reproduction. Species with high competitiveness go extinct due to their corresponding low colonization potential.

Our results show thus that specialist and generalist species can either invest in reproduction or in competition, except in the condition of high disturbance rate with limited dispersal, where investment in reproduction is critical for survival. Our results therefore contrast with the common conclusion that specialist species benefit from 'K-strategy' characteristics 
such as high competitive ability, while generalists are more associated with 'r-strategy' characteristics such as low competitive ability (Southwood 1988). We also find that competition and reproductive investment play less of an important role in specialist-generalist coexistence and in the emergence of trait strategies, when compared to the fundamental role of dispersal.

\section{Strategies involving specialisation, dispersal and competitiveness or reproductive investment}

In agreement with the results obtained by Ronce et al. (2000b), no global association was observed between dispersal and competitiveness/reproductive investment when dispersal varies along with competitiveness and reproductive investment. However, interestingly, an association between these traits emerges when species are grouped by specialisation levels.

When no disturbance occurs and there is no generation overlap, recruitment is high and homogeneous, favouring species with both reduced dispersal and increased reproductive investment (Ronce and Olivieri 1997). In contrast, when disturbance rate and generation overlap both occur, generalist species disperse effectively and invest greatly in reproduction. This situation is similar to gap creation in rain forests or coral reefs, leading to a "fugitive species syndrome" (Connell 1978, Tilman 1994).

Our results demonstrate that there is not a unique association between specialisation, dispersal ability and reproductive investment. These associations are instead driven by recruitment opportunities that are determined by disturbance rate and generation overlap. Similar results were obtained by Venable and Brown (1988), who show that the relative importance of seed traits (e.g. size, dispersal, dormancy) depends on dispersal limitation, spatial and temporal autocorrelation and disturbance.

\section{Model discussion and perspectives}

The approach adopted in our model consisted of generating species rich metacommunities in which each species has random trait values. We subsequently observed the selection of strategies that allowed species survival. This corresponds to community assembly processes, which are adopted less often than genetic approaches (but see for example Kallimanis et al. 2006, Devictor and Robert 2009). During simulations, traits did not evolve and the invasion of new or formerly extinct species was not allowed. We assumed that the time scale of the processes studied did not allow for trait evolution, as, our system is meant to mimic, for example, the colonisation of a novel habitat by competing species or a community experiencing a rapid change of its environment, as expected with anthropogenic changes. The use of a simulation model was necessary given the multiplicity of the traits considered and number of processes involved, as such a system would not be analytically tractable. Simulations also allow a thorough characterization of the successful strategies under different conditions. Finally, our simulation framework complements common models used to test theoretical metacommunity paradigms (reviewed by Logue et al. 2011).

Here, we considered 50 replicates of simulations, each starting with 100 species. This translated into a random sample of $100 \times 50$ combinations of trait values. This procedure allowed for the exploration of a large range of trait values. The number of species considered in our simulation set up is representative of what is generally observed in natural communities. Indeed, species richness is generally around 20-40 species and rarely goes beyond 100 species in temperate grasslands and forests (Proulx and Mazumder 1998, Klimek et al. 2007, Morin et al. 2011, Axmanova et al. 2012). Accounting for a larger number of initial species does not qualitatively change the results (see results for 1000 species in the Supplementary material Appendix 2). However, it has the consequence of generating many highly specialised species that occupy the whole range of environmental space. This process drives generalist species to extinction; we therefore cannot effectively describe strategies for generalist species under this condition. When a reduced number of species is considered (see results for 10 species in the Supplementary material Appendix 2), the resulting metacommunity poorly represents the potential diversity of strategies and combine few trait values. In this case, competition is very low and almost all species are maintained in the metacommunity, and strategies are thus not easy to identify.

In our simulations, the associations found between niche breadth and other traits are generally quantitatively but not qualitatively impacted by spatial autocorrelation (see Supplementary material Appendix 3). The main differences are observed in the presence of disturbance. We expect a more drastic effect if this environmental autocorrelation is associated with autocorrelation in disturbance (as predicted for fire, hurricane, or drought). Indeed it has been shown, in a metapopulation context, that the effect of autocorrelated disturbance can interact with habitat configuration (Vuilleumier et al. 2007). In this situation, specialist species might be driven to extinction by aggregated disturbance in their clustered habitats while generalist species might find refuge in more type of habitats, allowing re-colonisation following disturbance events.

The exact form of a tradeoff can influence the species coexistence outcome (Levins 1968, Yu and Wilson 2001, Levine and Rees 2002, Nurmi and Parvinen 2008). Our simulations considered two tradeoffs: one with habitat specialisation, and the other with investment in competition and reproduction. The specialisation tradeoff considered here is based on the species growth rate, which might be more detrimental to generalist species than specialist ones (Nurmi and Parvinen 2008). The tradeoff between competition and reproduction modelled here was inspired by the well-documented tradeoff between number and size of flowering plants' seed (Leishman 2001). While this tradeoff has been shown to promote coexistence (Tilman 1994, Yu and Wilson 2001), its role in shaping natural communities' composition, and its consequences in terms of species performance, have often been questioned (Leishman 2001, Moles and Westoby 2006, Agrawal et al. 2010). Moreover, tradeoffs are expected to vary among species and depend on the environments experienced by the species (Agrawal et al. 2010). How such variability impacts species' coexistence in a community remain to be investigated.

Our results show that dispersal is a critical factor in shaping trait associations in metacommunities. To gain further insights on its impact on community composition, 
we suggest that dispersal rate and dispersal distance should be considered as two independent factors. We expect that specialist species would benefit from rare, long distance dispersal events but do not require a high dispersal rate.

Specialist species are currently declining worldwide (Warren et al. 2001, Munday 2004, Clavel et al. 2011). However, our results demonstrate that specialists could be successful even in the presence of disturbance. This counterintuitive result could be explained by the large availability of habitats for specialist species in our study, while in natural ecosystems, specialist species suffer from important habitat loss. The impact of habitat loss on the coexistence of specialist and generalist species, and on the association of specialisation with the other traits, especially dispersal ability, would thus deserve further investigation.

Very few experimental investigations on the association of specialisation and other life history traits exist. However, recently, two experimental studies on trait co-variation with specialisation reported interesting and unexpected results. First, Khokhlova et al. (2014) demonstrated, using fleas, that stronger tradeoffs between quantity and quality of offspring are found for generalist species compared to specialist ones. Second, Condon et al. (2014) showed, using Drosophila melanogaster, that specialist and generalists genotypes do not necessary evolve in spatially constant and variable environments, respectively. We hope that our results will stimulate further experimental tests.

\section{Conclusions}

Our results show that distinct ecological strategies emerge principally for generalist species, whereas specialist species have much larger ranges of trait values and associations. The drivers of strategies are disturbance, in interaction with species dispersal ability and local recruitment opportunities. In natural habitats, these processes are strongly impacted by anthropogenic changes, such as increased disturbance, habitat fragmentation and habitat loss. This could explain the important changes in the degree of specialisation seen in natural communities (Warren et al. 2001, Munday 2004, Clavel et al. 2011). A better understanding of the characteristics linked to specialisation is therefore essential, as specialists are currently declining worldwide. In particular, the association of specialisation with other life-history traits would benefit from further empirical evidence.

Acknowledgements - The authors thank the editor whose comments and suggestions greatly improved the manuscript. We would also like to thank Sergio Rasmann for his improving comments and Kristen Irwin for a careful reading of the manuscript. This work was funded by the Swiss National Science Foundation grants no. 31003A-112511/2 to LB and PZ00P3-139421/1 and PMPDP3 158381 to SV, and by the FBM Interdisciplinary Grant of the Univ. of Lausanne to SV.

\section{References}

Adler, P. B. et al. 2014. Functional traits explain variation in plant life history strategies. - Proc. Natl Acad. Sci. USA 111: 740-745.
Agrawal, A. A. et al. 2010. Tradeoffs and adaptive negative correlations in evolutionary ecology. - In: Bell, M. A. (eds), Evolution after darwin: the first 150 years. Sinauer, pp. 243-268.

Axmanova, I. et al. 2012. The species richness-productivity relationship in the herb layer of European deciduous forests. - Global Ecol. Biogeogr. 21: 657-667.

Bonte, D. et al. 2012. Costs of dispersal. - Biol. Rev. Camb. Phil. Soc. 87: 290-312.

Borges, R. M. 2009. Phenotypic plasticity and longevity in plants and animals: cause and effect? - J. Biosci. 34: 605-611.

Brown, J. S. and Pavlovic, N. B. 1992. Evolution in heterogeneous environments - effects of migration on habitat specialisation. - Evol. Ecol. 6: 360-382.

Büchi, L. and Vuilleumier, S. 2014. Coexistence of specialist and generalist species is shaped by dispersal and environmental factors. - Am. Nat. 183: 612-624.

Büchi, L. and Vuilleumier, S. 2015. Data from: Ecological strategies in stable and disturbed environments depend on species specialisations. - Dryad Digital Repository, <http://dx.doi. org/10.5061/dryad.ms738>.

Büchi, L. et al. 2009. The influence of environmental spatial structure on the life-history traits and diversity of species in a metacommunity. - Ecol. Modell. 220: 2857-2864.

Buoro, M. and Carlson, S. M. 2014. Life-history syndromes: integrating dispersal through space and time. - Ecol. Lett. 17: 756-767.

Clavel, J. et al. 2011. Worldwide decline of specialist species: toward a global functional homogenization? - Front. Ecol. Environ. 9: 222-228.

Condon, C. et al. 2014. Temporal variation favors the evolution of generalists in experimental populations of Drosophila melanogaster. - Evolution 68: 720-728.

Connell, J. H. 1978. Diversity in tropical rain forests and coral reefs. - Science 199: 1302-1310.

Dahirel, M. et al. 2015. Movement propensity and ability correlate with ecological specialization in European land snails: comparative analysis of a dispersal syndrome. - J. Anim. Ecol. 84: 228-238.

Débarre, F. and Gandon, S. 2010. Evolution of specialization in a spatially continuous environment. - J. Evol. Biol. 23: 1090-1099.

Devictor, V. and Robert, A. 2009. Measuring community responses to large-scale disturbance in conservation biogeography. - Divers. Distrib. 15: 122-130.

Devictor, V. et al. 2008. Distribution of specialist and generalist species along spatial gradients of habitat disturbance and fragmentation. - Oikos 117: 507-514.

Dynesius, M. and Jansson, R. 2000. Evolutionary consequences of changes in species' geographical distributions driven by Milankovitch climate oscillations. - Proc. Natl Acad. Sci. USA 97: 9115-9120.

Fridley, J. D. et al. 2007. Co-occurrence based assessment of habitat generalists and specialists: a new approach for the measurement of niche width. - J. Ecol. 95: 707-722.

Futuyma, D. J. and Moreno, G. 1988. The evolution of ecological specialisation. - Annu. Rev. Ecol. Syst. 19: 207-233.

Gandon, S. and Michalakis, Y. 1999. Evolutionarily stable dispersal rate in a metapopulation with extinctions and kin competition. - J. Theor. Biol. 199: 275-290.

Goovaerts, P. 1998. Impact of the simulation algorithm magnitude of ergodic fluctuations and number of realizations on the spaces of uncertainty of flow predictions no. 11. - Stanford University.

Grime, J. P. 1977. Evidence for existence of three primary strategies in plants and its relevance to ecological and evolutionary theory. - Am. Nat. 111: 1169-1194.

Grime, J. P. and Pierce, S. 2012. The evolutionary strategies that shape ecosystems. - Wiley. 
Hastings, A. 1983. Can spatial variation alone lead to selection for dispersal. - Theor. Popul. Biol. 24: 244-251.

Jakobsson, A. and Eriksson, O. 2000. A comparative study of seed number, seed size, seedling size and recruitment in grassland plants. - Oikos 88: 494-502.

Kallimanis, A. S. et al. 2006. Patchy disturbance favours longer dispersal distance. - Evol. Ecol. Res. 8: 529-541.

Khokhlova, I. S. et al. 2014. A tradeoff between quantity and quality of offspring in haematophagous ectoparasites: the effect of the level of specialisation. - J. Anim. Ecol. 83: 397-405.

Kisdi, E. 2002. Dispersal: risk spreading versus local adaptation. - Am. Nat. 159: 579-596.

Klimek, S. et al. 2007. Plant species richness and composition in managed grasslands: the relative importance of field management and environmental factors. - Biol. Conserv. 134: 559-570.

Leishman, M. R. 2001. Does the seed size/number tradeoff model determine plant community structure? An assessment of the model mechanisms and their generality. - Oikos 93: 294-302.

Levin, S. A. and Muller-Landau, H. C. 2000. The evolution of dispersal and seed size in plant communities. - Evol. Ecol. Res. 2: 409-435.

Levine, J. M. and Rees, M. 2002. Coexistence and relative abundance in annual plant assemblages: the roles of competition and colonization. - Am. Nat. 160: 452-467.

Levins, R. 1968. Evolution in changing environments. - Princeton Univ. Press.

Logue, J. B. et al. 2011. Empirical approaches to metacommunities: a review and comparison with theory. - Trends Ecol. Evol. 26: 482-491.

MacArthur, R. H. and Wilson, E. O. 1967. The theory of island biogeography. - Princeton Univ. Press.

Marvier, M. et al. 2004. Habitat destruction, fragmentation, and disturbance promote invasion by habitat generalists in a multispecies metapopulation. - Risk Anal. 24: 869-878.

Moles, A. T. and Westoby, M. 2006. Seed size and plant strategy across the whole life cycle. - Oikos 113: 91-105.

Morin, X. et al. 2011. Tree species richness promotes productivity in temperate forests through strong complementarity between species. - Ecol. Lett. 14: 1211-1219.

Munday, P. L. 2004. Habitat loss, resource specialisation, and extinction on coral reefs. - Global Change Biol. 10: 1642-1647.

Nilsson, A. N. et al. 1993. Macroptery in altitudinal specialists versus brachyptery in generalists - a paradox of alpine scandinavian carabid beetles (coleoptera: Carabidae). - J. Biogeogr. 20: 227-234.

Nurmi, T. and Parvinen, K. 2008. On the evolution of specialisation with a mechanistic underpinning in structured metapopulations. - Theor. Popul. Biol. 73: 222-243.

Nurmi, T. and Parvinen, K. 2011. Joint evolution of specialization and dispersal in structured metapopulations. - J. Theor. Biol. 275: 78-92.

Parvinen, K. and Egas, M. 2004. Dispersal and the evolution of specialisation in a two-habitat type metapopulation. - Theor. Popul. Biol. 66: 233-248.

Pianka, E. R. 1970. On r-selection and k-selection. - Am. Nat. 104: 592-597.

Supplementary material (available online as Appendix oik02915 at <www.oikosjournal.org/appendix/oik-02915>). Appendix 1-4.
Poisot, T. et al. 2011. A conceptual framework for the evolution of ecological specialisation. - Ecol. Lett. 14: 841-851.

Proulx, M. and Mazumder, A. 1998. Reversal of grazing impact on plant species richness in nutrient-poor vs nutrient-rich ecosystems. - Ecology 79: 2581-2592.

Ravigné, V. et al. 2009. Live where you thrive: joint evolution of habitat choice and local adaptation facilitates specialisation and promotes diversity. - Am. Nat. 174: E141-E169.

Reich, P. B. 2014. The world-wide 'fast-slow' plant economics spectrum: a traits manifesto. - J. Ecol. 102: 275-301.

Reich, P. B. et al. 2003. The evolution of plant functional variation: traits, spectra and strategies. - Int. J. Plant Sci. 164: S143-S164.

Reif, J. et al. 2015. Linking habitat specialization with species' traits in European birds. - Oikos 125: 405-413.

Ronce, O. and Olivieri, I. 1997. Evolution of reproductive effort in a metapopulation with local extinctions and ecological succession. - Am. Nat. 150: 220-249.

Ronce, O. and Kirkpatrick, M. 2001. When sources become sinks: migrational meltdown in heterogeneous habitats. - Evolution 55: $1520-1531$.

Ronce, O. et al. 2000a. Evolutionarily stable dispersal rates do not always increase with local extinction rates. - Am. Nat. 155: 485-496.

Ronce, O. et al. 2000b. Landscape dynamics and evolution of colonizer syndromes: interactions between reproductive effort and dispersal in a metapopulation. - Evol. Ecol. 14: 233-260.

Southwood, T. R. E. 1988. Tactics, strategies and templets. - Oikos 52: 3-18.

Stevens, V. M. et al. 2014. A comparative analysis of dispersal syndromes in terrestrial and semi-terrestrial animals. - Ecol. Lett. 17: 1039-1052.

Tilman, D. 1994. Competition and biodiversity in spatially structured habitats. - Ecology 75: 2-16.

Venable, D. L. and Brown, J. S. 1988. The selective interactions of dispersal, dormancy and seed size as adaptations for reducing risk in variable environment. - Am. Nat. 131: 360-384.

Verberk, W. et al. 2010. Explaining abundance-occupancy relationships in specialists and generalists: a case study on aquatic macroinvertebrates in standing waters. - J. Anim. Ecol. 79: 589-601.

Vuilleumier, S. et al. 2007. How patch configuration affects the impact of disturbances on metapopulation persistence. - Theor. Popul. Biol. 72: 77-85.

Warren, M. S. et al. 2001. Rapid responses of british butterflies to opposing forces of climate and habitat change. - Nature 414: 65-69.

Westoby, M. 1998. A leaf-height-seed (LHS) plant ecology strategy scheme. - Plant Soil 199: 213-227

Westoby, M. et al. 1992. Comparative evolutionary ecology of seed size. - Trends Ecol. Evol. 7: 368-372.

Westoby, M. et al. 2002. Plant ecological strategies: some leading dimensions of variation between species. - Annu. Rev. Ecol. Syst. 33: 125-159.

Wright, I. J. et al. 2004. The worldwide leaf economics spectrum. - Nature 428: 821-827.

Yu, D. W. and Wilson, H. B. 2001. The competition-colonization tradeoff is dead; long live the competition-colonization tradeoff. - Am. Nat. 158: 49-63. 